\title{
Investigación en Diseño en la Universidad de Palermo
}

Memoria de Investigación en Diseño 2015-2020 (Volumen 1 y 2)

Es un orgullo compartir los dos Volúmenes de la presente Memoria de Investigación en Diseño. Con esta Memoria nace el Instituto de Investigación en Diseño ${ }^{1}$ de nuestra Facultad.

La Facultad de Diseño y Comunicación nació, junto con la Universidad de Palermo, en el año 1991. En el año 2000 comenzó su Plan de Investigación que hasta el 2010 se organizó en el Centro de Estudios en Diseño y Comunicación. Desde el año 2011 hasta el año 2020 el Centro se transformó en el Programa de Investigación en Diseño. Y en ocasión de esta Memoria el Programa se transformó en el Instituto de Investigación en Diseño.

La presente Memoria cubre el desarrollo del Programa de Investigación en el período 2015-2020 que corresponde al denominado Ciclo B del Plan de Investigación de la Facultad de Diseño y Comunicación que comenzó en el año 2020 y fue consolidándose en sus 20 años de desarrollo como se relata más adelante en este artículo.

El Programa de Investigación se organiza en grandes Líneas de Investigación y cada una de estas Líneas agrupa varios Proyectos. Así cada Proyecto pertenece a una Línea de Investigación. En el Volumen 1 se incluyen los correspondientes a las Líneas 1 a 9 (27 Proyectos) y en el Volumen 2 se incluyen los correspondientes a las Líneas 10 a 18 (35 Proyectos).

El Programa de Investigación en Diseño estableció la formalidad que a todo Proyecto se lo considera finalizado cuando publica sus resultados en una edición de Cuadernos del Centro de Estudios en Diseño y Comunicación.

Por lo tanto todos los Proyectos incluidos en esta Memoria son Proyectos concluidos con su respectiva edición en Cuadernos que documenta y comparte sus aportes de investigación y reflexión. No se incluyen Proyectos en desarrollo, que integrarán la próxima Memoria. Asimismo todos los Proyectos de esta Memoria han sido desarrollados en el marco del Programa de Investigación de la Facultad de Diseño y Comunicación de la Universidad de Palermo. Muchos de ellos en colaboración con otras instituciones nacionales e internacionales (44 Proyectos).

1. El sitio del Instituto de Investigación en Diseño está disponible en https://www.palermo.edu/dyc/instituto_investigacion/index.html 
Cada Proyecto se presenta con su denominación, su dirección académica y las instituciones que lo desarrollaron. Cada Proyecto tiene un código numérico único que lo identifica y lo vincula a la Línea de pertenencia (ej. 1.2: el primer número corresponde a la Línea y el segundo al Proyecto).

Luego está el resumen del Proyecto con sus palabras clave en español, inglés y portugués. Se presentan brevemente sus objetivos y su vinculación con la Línea de Investigación respectiva. Se detallan los productos y resultados de cada Proyecto organizados en: a) Publicaciones y b) Presentaciones en Congresos, Coloquios y Plenarios organizados por la Facultad de Diseño y Comunicación. Por razones de alcance de la presente Memoria no se incluye otra información.

En el ítem Publicaciones se presenta la edición del Cuaderno que documenta los resultados del Proyecto con el detalle de cada uno de los artículos con sus autores, incluidos en dicha edición, con el paginado respectivo.

En el ítem Congresos, Coloquios y Plenarios se documentan las presentaciones que los investigadores de cada proyecto hicieron en las diferentes ediciones del Congreso Latinoamericano de Enseñanza de Diseño, del Coloquio Internacional de Investigadores en Diseño y del Plenario de Directores de Líneas y Proyectos de Investigación.

\section{Del Centro al Programa y del Programa al Instituto}

El Programa de Investigación en Diseño de la Facultad de Diseño y Comunicación de la Universidad de Palermo produce, organiza y difunde parte de la producción original de conocimiento de la Unidad Académica en este campo disciplinar, articulada con otros aportes en este campo que surgen de las carreras de grado, los posgrados que dicta (Doctorado en Diseño y Maestría en Gestión del Diseño ${ }^{2}$ ) y la plataforma de proyección internacional (publicaciones, congresos, acuerdos y redes) que construyó la unidad académica.

El Programa, concreta uno de los objetivos centrales de la misión de la Facultad respecto al fomento, consolidación e integración curricular de la investigación interdisciplinaria en Diseño, entendiendo las necesidades y contribuyendo con los requerimientos en este campo de las instituciones académicas del país y la región.

2. La información del Doctorado en Diseño y la Maestría en Gestión del Diseño se encuentran disponibles en https://www.palermo.edu/dyc/posgrados/index.html 
El alcance regional y la proyección internacional del Programa de Investigación en Diseño es posible gracias al desarrollo sistemático y sostenido, desde su creación en el año 1990, de un conjunto de acciones concebidas y desarrolladas por la Facultad de Diseño y Comunicación, tales como la organización de Congresos y Coloquios, el impulso y la coordinación de redes colaborativas, la firma de acuerdos con instituciones de todo el mundo que integran el Programa de Colaboración para el Desarrollo Académico de Diseño y Comunicación, la conformación internacional de sus posgrados y la realización de proyectos de investigación interinstitucionales con la publicación de sus resultados en forma conjunta, entre otras acciones (Puede consultarse: Investigación en Diseño en Palermo / Publicaciones con Resultados de Investigaciones con Instituciones Internacionales y Nacionales / 2005-2020).

Esta articulación de factores, impulsados estratégicamente desde la creación de la Universidad de Palermo, ha instalado a la Facultad de Diseño y Comunicación como un indiscutido referente de calidad académica tal como lo testimonia la calificación obtenida en el Ranking Internacional QS ${ }^{4}$ (World University Rankings QS by Subject) en la categoría Diseño en los últimos siete años.

Desde la creación de este Ranking en 2015 (y en las sucesivas ediciones 2016, 2017, 2018, 2019, 2020 y 2021) la Universidad de Palermo estuvo ubicada, por su calidad educativa, entre las 100 universidades mejor evaluadas del mundo en la disciplina Diseño y, en tal sentido, ha sido calificada como la mejor universidad privada de Argentina.

La constitución del Programa, como propuesta articulada de investigación, es uno de los grandes logros, estructurales y estratégicos, logrados por la Facultad. El Programa de Investigación en Diseño de la Facultad de Diseño y Comunicación de la Universidad de Palermo se diseñó y conceptualizó en el año 2010 a partir de la experiencia y los logros del período 2001-2010 del entonces Centro de Estudios en Diseño y Comunicación. El Programa, en su estructura actual, comenzó a desarrollarse en el año 2011 en ocasión de la presentación a la Comisión Nacional de Evaluación y Acreditación Universitaria (CONEAU) de los Proyectos del Doctorado en Diseño y de la Maestría en Gestión del Diseño que, previa acreditación, comenzaron a dictarse en el año 2014.

El Programa planifica y organiza su crecimiento en grandes Ciclos de cinco años de duración cada uno. Hasta el presente, se han desarrollado el Ciclo A: del 2011 al 2015,

\footnotetext{
3. La publicación -Investigación en Diseño en Palermo / Publicaciones con Resultados de Investigaciones con Instituciones Internacionales y Nacionales / 2005-2020- está disponible en: https://www.palermo.edu/ dyc/investigacion_desarrollo_diseno_latino/Investigacion_en_Diseno_en_Palermo.pdf

4. El Ranking Internacional QS Diseño y Comunicación, El mejor Diseño esta en Palermo, se encuentra disponible en: https://www.palermo.edu/dyc/elmejordiseno/index.html
} 
y el Ciclo B: del 2016 al 2020. Para cada uno de ellos se plantearon objetivos específicos articulados con el plan integral de desarrollo de la Facultad en su conjunto.

La Facultad de Diseño y Comunicación nace en el año 1990 simultáneamente con la creación de la Universidad de Palermo. Desde su creación, entendió la necesidad de estimular la investigación de su campo en sus carreras, incorporando y formando a sus docentes y estudiantes y organizando la difusión de resultados a través de publicaciones y espacios académicos de debate y reflexión con proyección regional.

\section{1-2010: Centro de Estudios en Diseño y Comunicación}

Así, entre el año 2000 y el año 2010 la Facultad impulsó investigaciones que, si bien cumplían con los estándares académicos requeridos, se desarrollaban de manera aislada, constituyendo una etapa exploratoria en el campo de la investigación en Diseño. En aquella primera etapa fundacional, cuyas acciones estaban enmarcadas en el entonces Centro de Estudios en Diseño y Comunicación, se conformaron los imprescindibles primeros pasos de un proceso ascendente en la producción de conocimiento. En dicho período, se crearon y articularon diversas actividades y comenzó la formación de recursos humanos ávidos por investigar y diligenciar sus acciones en el marco institucional de la Facultad. Uno de los principales logros de ese entonces fue incentivar y formar al claustro docente en las tareas de proponer y planificar, de promover y sostener prácticas y proyectos de investigación.

En el año 2000, la Facultad comenzó a editar la publicación académica Cuadernos del Centro de Estudios en Diseño y Comunicación ${ }^{5}$ que, en un proceso de consolidación y reconocimiento nacional e internacional que requirió de varios años, fue convirtiéndose en el principal vehículo académico de documentación y difusión de los resultados de las investigaciones realizadas.

En el año 2007, el entonces Ministerio de Ciencia, Tecnología e Innovación de la República Argentina, la reconoció incorporándola al Núcleo Básico de Revistas Científicas Argentinas (NBR), que es un proyecto del Consejo Nacional de Investigaciones Científicas y Técnicas (CONICET) de la República Argentina en la Categoría Ciencias Sociales y Humanidades. Desde ese año, la publicación permanece en este NBR mejorando sus sucesivas evaluaciones hasta el presente. En la actualidad Cuadernos del Centro de Estudios en Diseño y Comunicación tiene una edición papel (ISSN 1668-0227), una digital (ISSN Online: 1853-3523) y DOI: https://doi.org/. Además, esta publicación está

5. Los Cuadernos del Centro de Estudios en Diseño y Comunicación, están disponibles en: https://fido. palermo.edu/servicios_dyc/publicacionesdc/cuadernos/index.php 
indizada en varios y prestigiosos repositorios bibliográficos tales como Scielo, Latindex, Dialnet y Epsco, y sus contenidos están disponibles on line, en forma gratuita ${ }^{6}$.

Es importante señalar que en el año 2002 la Unidad Académica comenzó a dictar la Maestría en Diseño, y continuó, en forma ininterrumpida con su desarrollo hasta 2014. A partir de aquel entonces, se transformó en Maestría en Gestión del Diseño que se dicta hasta el presente. Aquella, fue la primera Maestría en este campo en la Argentina, una de las pioneras en la región y uno de los antecedentes de la creación del Doctorado en Diseño en la Unidad Académica.

En la primera década del siglo XXI, y puntualmente en julio de 2006, comenzó a realizarse la Semana Internacional de Diseño en Palermo ${ }^{7}$ (antes se denominaba Encuentro Latinoamericano de Diseño) que continúa realizándose anualmente hasta el presente. Al año siguiente, 2007, se conformó el Foro de Escuelas de Diseño ${ }^{8}$, que actualmente es una red con 400 instituciones educativas del continente, que funciona activamente en el campo del diseño y que coordina la Universidad de Palermo.

El Foro, cuyos plenarios sesionan anualmente en la Universidad de Palermo, creó en el año 2010 el Congreso Latinoamericano de Enseñanza del Diseño ${ }^{9}$, cuyas reflexiones, ponencias, artículos y comunicaciones se difunden a través de la publicación académica internacional Actas de Diseño ${ }^{10}$ (ISSN 1850-2032; ISSN en línea: 2591-3735).

La década 2000-2010 fue muy fructífera en el establecimiento de un conjunto de iniciativas (Publicaciones, Congreso, Foro, Encuentro Latinoamericano, Maestría y otras) que se presentaron en los párrafos anteriores y que fueron sostenidas en el tiempo. Las mismas construyeron la base institucional para instalar, organizar y desarrollar exitosamente en la siguiente década 2010-2020 un Programa de Investigación en Diseño integral, participativo y de proyección internacional en la Unidad Académica.

El Programa de Investigación en Diseño que continua, enriqueciendo y profundizando, las actividades desarrolladas en el periodo 2001-2010 por el Centro de Estudios en Diseño

6. La Serie Cuadernos está disponible online, en forma gratuita en https://fido.palermo.edu/servicios_dyc/ publicacionesdc/cuadernos/index.php

7. El sitio de La Semana Internacional de Diseño en Palermo está disponible en https://fido.palermo.edu/ servicios_dyc/semanavirtualinternacionaldediseno/index.php

8. La información acerca del Foro de Escuelas de Diseño está disponible en https://www.palermo.edu/dyc/ foro_congreso/index.html

9. El sitio del Congreso Latinoamericano de Enseñanza del Diseño está disponible en https://www.palermo. edu/dyc/congreso-latino

10. La publicación Actas de Diseño se encuentra disponible en https://fido.palermo.edu/servicios_dyc/ publicacionesdc/actas_de_diseno/index.php 
y Comunicación, es concebido como un sistema integrado que articula, potenciándolas entre sí, un conjunto de acciones institucionales. El Programa, fue concebido, organizado e impulsado al cumplir la unidad académica 20 años de vida, en ocasión de la presentación del Proyecto de Creación del Doctorado en Diseño y de la Maestría en Gestión del Diseño en el año 2011.

\section{1-2015: Programa de Investigación en Diseño (Ciclo A)}

A través del proceso desarrollado en el Ciclo A (2011-2015) se terminó de estructurar el Programa como un sistema que se organiza en Líneas de Investigación plurianuales dirigidas, cada una, por un investigador de la Facultad de Diseño y Comunicación, muchos de ellos vinculados a sus Posgrados (Doctorado en Diseño y Maestría en Gestión del Diseño).

A su vez en cada una de las Líneas se desarrollan Proyectos de Investigación (Ver Indice de Líneas y Proyectos del Programa de Investigación Pp. 11 y 17). Cada uno de estos Proyectos tiene sus propios objetivos, alcances, duración y, cuando corresponde, acuerdos interinstitucionales que lo enmarcan y proyectan regional y/o internacionalmente. Cada Proyecto tiene un coordinador de la Facultad de Diseño y Comunicación, y en los casos de acuerdos interinstitucionales comparte la coordinación con el responsable designado por la contraparte. El Programa está guiado por una visión amplia que asume una concepción multidisciplinaria del Diseño que se enriquece al incorporar marcos conceptuales, enfoques metodológicos y fuentes documentales de otras disciplinas proyectuales, sociales y humanas.

Es importante señalar que en el 2014, enmarcado en este Ciclo A del Programa, comenzaron a dictarse el Doctorado en Diseño y la Maestría en Gestión del Diseño (continuidad de la Maestría en Diseño, que se dictó ininterrumpidamente desde 2002 hasta el 2013 inclusive), ambos con recomendación favorable por la Comisión Nacional de Evaluación y Acreditación Universitaria (CONEAU) por Dictámenes del 18/11/13 (Sesión $\mathrm{N}^{\circ} 389$ ) y del 3/12/12 (Sesión $\mathrm{N}^{\circ} 367$ ) respectivamente.

Estos posgrados académicos fueron el ámbito para el impulso a las nuevas investigaciones a través del liderazgo de sus docentes que coordinan Proyectos en el Programa de Investigación y cuentan con la participación activa de los estudiantes en los procesos conducentes a sus Tesis respectivas.

En este período se formalizó el Programa de Colaboración para el Desarrollo Académico de Diseño y Comunicación, que reúne a todas las instituciones universitarias con las que la Facultad de Diseño y Comunicación ha firmado acuerdos de cooperación y desarrollo académico. 
Entre estos acuerdos están aquellos que implican el desarrollo de proyectos de investigación, en forma conjunta entre ambas instituciones como los firmados con Parsons, The New School for Design (Nueva York, Estados Unidos), School of Design at Carnegie Mellon University (Estados Unidos), University of Cambridge, Centre of Latin American Studies (Inglaterra), Instituto Politécnico de Milán (Italia), Universita Di Bologna (Italia), Universidad Nacional Autónoma de México (México), Escola de Artes, Ciencias e Humanidades y Estacio Da Sal (San Pablo, Brasil), Universidade Estadual de Minas Gerais (Brasil) y el Instituto Gino Germani de la Universidad de Buenos Aires (Argentina), entre otros (Ver Índice de Instituciones con Investigaciones).

En el año 2015 se creó el Ranking internacional QS que califica la calidad de las universidades por disciplina (World University Rankings QS by Subject). La Universidad de Palermo estuvo ubicada, por su calidad educativa, entre las 50 universidades mejor evaluadas del mundo en la disciplina Diseño y, en tal sentido, ha sido calificada como la mejor universidad privada de Argentina. Evaluación que conservó y mejoró en las sucesivas ediciones. En la última edición del mismo (2019) avanzó hasta ocupar el segundo puesto en América Latina.

En efecto, en esos 5 años se han plasmado diversas estrategias de consolidación de los equipos académicos, de formación de recursos humanos y de financiamiento institucional que contribuyeron a darle continuidad, sostenimiento y proyección a las investigaciones desarrolladas.

\section{6-2020: Programa de Investigación en Diseño (Ciclo B)}

En el desarrollo del Ciclo B (2016-2020), docentes del Doctorado en Diseño y/o de la Maestría en Gestión del Diseño, que son investigadores del Programa vincularon e incorporaron activamente las investigaciones en curso de sus maestrandos y/o doctorandos con sus diferentes Proyectos de Investigación.

El estímulo a la cultura de la investigación y a la constitución de una comunidad de investigadores en Diseño se refuerza con la realización de los Foros de Investigación. Cada seis meses los proyectos de investigación que llevan adelante los doctorandos son presentados en el Foro de Investigación del Doctorado y los maestrandos hacen lo mismo, anualmente, en su Foro de Investigación de la Maestría. En cada Foro participa la comunidad académica del posgrado respectivo y se documenta en una edición de la 
publicación Escritos en la Facultad ${ }^{11}$ (ISSN 1669-2306; ISSN en línea: 2591-3743) en el que se difunden los resúmenes de todos los proyectos con referencia a sus autores y su nivel de avance.

Así, se constituyeron los posgrados como espacios de socialización y retroalimentación de investigadores formados y en formación, funcionando como una verdadera usina de construcción colaborativa de conocimientos y de empoderamiento de las nuevas generaciones de investigadores en Diseño como puede comprobarse en la calidad de las Tesis presentadas y aprobadas ${ }^{12}$.

En el transcurso del Ciclo B (2016-2020) se terminó de conformar la plataforma institucional de difusión, comunicación, documentación y registro que incluye la publicación de los resultados en "Cuadernos del Centro de Estudios en Diseño y Comunicación” y su presentación en las ediciones del Congreso Latinoamericano de Enseñanza del Diseño, en los Coloquios Internacionales de Investigadores en Diseño y en los Plenarios de Directores de Investigación, independiente a aquellas acciones que realizan sus investigadores en forma personal y, cuando corresponde, las instituciones que comparten la coordinación. La Presente Memoria, en sus dos volúmenes, recopila los resultados de los Proyectos de Investigación realizados entre los años 2016 y 2020, correspondiente al Ciclo B del Programa de Investigación en Diseño.

En el año 2016 se creó el Coloquio Internacional de Investigadores en Diseño ${ }^{13}$ que en el 2020 realizó su V edición. En el Coloquio, los investigadores de la Unidad Académica y de las instituciones internacionales que participan del Programa de Investigación en Diseño presentan en comisiones los resultados alcanzados, y publicados, resultado del trabajo académico colaborativo desarrollado durante el año en cada uno de sus Proyectos y exponen sus planes para el nuevo período convocando a participar en los mismos.

En el año 2017 se realizó el primer Plenario de Directores de Proyectos de Investigación del Programa ${ }^{14}$. Este Plenario, que se realiza anualmente, se constituyó en un espacio de

11. La publicación Escritos en la Facultad está disponible en https://fido.palermo.edu/servicios_dyc/ publicacionesdc/vista/publicaciones.php?id_publicacion $=5$

12. El Directorio de Tesis aprobadas de Maestria en Gestión del Diseño puede consultarse en: https://www. palermo.edu/dyc/maestria_diseno/tesis.html; y el de Doctorado en Diseño en https://www.palermo.edu/ dyc/doctorado_diseno/tesis.html

13. El sitio del Coloquio Internacional de Investigadores en Diseño está disponible en https://www. palermo.edu/dyc/coloquio_investigacion_y_desarrollo/index.html

14. El primer Plenario de Directores de Proyectos de Investigación del Programa puede consultarse en https://www.palermo.edu/dyc/investigacion_desarrollo_diseno_latino/1er_plenario.html 
análisis y balance, de debate, reflexión y planificación colaborativa del Programa entre todos los directores del mismo. Entre otros logros del Plenario, es importante mencionar que en el año 2018 se constituyó el Comité Externo de Evaluación del Programa ${ }^{15}$, y en al año 2020, se organizó la categorización de los docentes investigadores de la Unidad Académica.

En este Ciclo se sistematizó que los resultados de cada Proyecto del Programa de Investigación en Diseño se plasmen en la publicación académica internacional Cuadernos del Centro de Estudios en Diseño y Comunicación (ISSN papel 1668-0227, ISSN Online: 1853-3523) y DOI: https://doi.org/ que edita la Facultad de Diseño y Comunicación en forma ininterrumpida desde el año 2000 con Comité de Arbitraje internacional. Esta revista constituye uno de los espacios privilegiados para exponer y difundir los alcances de las investigaciones desarrolladas, la que se convirtió en un valioso medio de actualización, consulta, intercambio y debate de la comunidad internacional de investigadores y académicos. Hasta el presente se han publicado 120 ediciones de Cuadernos del Centro de Estudios. De ellos 62 son números de la colección realizados en forma conjunta con 50 instituciones pertenecientes a 13 países. (Ver Índice de Instituciones por países con Acuerdos Académicos internacionales en Pp. 19-31).

Cuando se trata de un Proyecto interinstitucional, se realiza la edición en forma conjunta con la contraparte. Estos resultados son evaluados por miembros del Comité Externo de Evaluación cuya constitución y funcionamiento es uno de los logros del período.

El Ciclo B (2016-2020), se caracteriza por ser la etapa de consolidación y crecimiento sostenido de todas las actividades que conforman el sistema del Programa de Investigación. Esta sistematización se articuló con la investigación colaborativa con numerosas instituciones de prestigio internacional ampliando, desde una perspectiva superadora, la proyección regional del Programa y la consolidación de equipos de investigación interinstitucionales e interdisciplinarios (Ver Listado de Líneas y Proyectos del Programa de Investigación en Págs 11 y 17).

Con la documentación y puesta a disposición de los resultados de los Proyectos de Investigación a toda la comunidad académica nacional e internacional, se constituyó un acervo bibliográfico original, que enriquece y consolida el corpus teórico y metodológico en, desde, para y por el Diseño que está disponible en forma libre y gratuita. ${ }^{16}$

15. La información sobre el Comité Externo de Evaluación del Programa se encuentra disponible en https://www.palermo.edu/dyc/programa_investigacion/comite_externo.html

16. Acervo bibliográfico original de la Facultad de Diseño y Comunicación disponible en https://fido. palermo.edu/servicios_dyc/publicacionesdc/cuadernos/index.php 
Toda la documentación del Programa de Investigación está disponible con acceso libre y gratuito en el sitio web del Programa. ${ }^{17}$

Como se plantea en el comienzo de este artículo con la publicación de la Memoria culmina el Ciclo B (2016-2020) y el Programa de Investigación en Diseño se transforma en el Instituto de Investigación en Diseño de la Universidad de Palermo inaugurando el Ciclo C (2021-2025) del Plan de Investigación de la Facultad de Diseño y Comunicación.

\section{Oscar Echevarría}

Decano

Facultad de Diseño y Comunicación

Universidad de Palermo, Argentina

17. El Programa de Investigación de la Facultad de Diseño y Comunicación está disponible en https://www. palermo.edu/dyc/programa_investigacion/ 